\title{
ON A CLASS OF LATTICE-ORDERED RINGS
}

\section{BARRON BRAINERD}

1. Introduction. It is the purpose of this paper to study a class of rings called $F$-rings. An $F$-ring $R$ is a $\sigma$-complete vector lattice (Birkhoff $[2$, p. 238]) which is, in addition, a commutative algebra with a unit 1 , satisfying the conditions

$$
1 \geqq 0 ; \quad x \geqq 0, y \geqq 0 \Rightarrow x y \geqq 0 ; \quad x \wedge 1=0 \Rightarrow x=0 .
$$

Here $\wedge$ denotes, as usual, the lattice operation greatest lower bound, and $x, y$ are elements of $R$. A bounded F-ring is an $F$-ring $\bar{R}$ such that each $x \in \bar{R}$ satisfies

$$
x \vee 0+(-x) \vee 0 \leqq \lambda \cdot 1
$$

for some real number $\lambda$, the symbol $\vee$ denoting the lattice least upper bound.

Any ring $R$ is regular [10] if for each $x \in R$ there is an $x^{0} \in R$ such that $x x^{0} x=x$. It is evident that every regular $F$-ring $R$ contains a maximal bounded sub- $F$-ring $\bar{R}$, the $F$-ring of all $x \in R$ satisfying equation (1.1). The relationship between a regular $F$-ring and its maximal bounded sub- $F$-ring is analogous to that between the ring of all continuous functions on a completely regular space $X$ and the ring of all bounded continuous functions on $X$. For example, it is shown in Theorem 3 that there is a one-to-one correspondence between the maximal ideals of $R$ and those of $\bar{R}$. (For the theory of rings of continuous functions, see [5] and [6].)

A maximal ideal $M$ of a ring $R$ is real [6] if the quotient ring $R-M$ is ring-isomorphic to the real field. An ideal $S$ of an $F$-ring $R$ is closed if $a_{n} \in S, n \geqq 1$ and $\bigvee_{n=1}^{\infty} a_{n} \in R$ imply $\bigvee_{n=1}^{\infty} a_{n} \in S$. It is proved in Theorems 5 and 6 that the closed maximal ideals of a regular $F$-ring are real and that there is a one-to-one correspondence between the closed maximal ideals of a regular $F$-ring $R$ and the closed maximal ideals of $\bar{R}$, the maximal bounded sub-F-ring of $R$.

It is a direct corollary of some results of Nakano [9, pp. 39, 212] that a bounded $F$-ring is ring- and lattice-isomorphic to the ring of all continuous functions on a compact Hausdorff space. Therefore every bounded $F$-ring is a semisimple real Banach algebra. "Real" is used here in the classical sense, that is, a partially ordered ring $R$ is

Presented to the Society, October 27, 1956; received by the editors April 30, 1955 and, in revised form, June 25, 1956. 
real provided every element $a \geqq \epsilon$ for some real $\epsilon>0$ has an inverse in $R$. (The notation $\epsilon$ is used in place of $\epsilon \cdot 1$.) An $F$-ring is called an $M$-ring if the intersection of all its real closed maximal ideals is the 0 -element. A nontrivial example of a regular $M$-ring is the ring of all continuous functions on a $P$-space [4]. If $\Phi$ is an abstract set and $\mathcal{R}$ is a $\sigma$-algebra of subsets of $\Phi$, then a real function $f(\xi)$ defined on $\Phi$ is said to be measurable or $(\Phi, \mathfrak{l})$-measurable if for each real $\lambda$ the set $\{\xi \mid f(\xi) \leqq \lambda\}$ belongs to $\mathfrak{R}$.

The main result of this paper (Theorem 7 ) is that any real $M$-ring $B$ is ring- and lattice-isomorphic to $B(\Omega, \mathfrak{A})$, an $F$-ring of $(\Omega, \mathfrak{A})$ measurable functions. Here $\Omega$ designates the set of real closed maximal ideals of $B$, and $\mathfrak{A}$ is a $\sigma$-algebra of subsets of $\Omega$ isomorphic to the Boolean algebra $I$ of idempotents of $B$. In addition (corollary to Theorem 8$)$, if $B$ is a regular, then $B(\Omega, \mathfrak{A})$ is the $F$-ring of all $(\Omega, \mathfrak{A})$ measurable functions.

2. Notation and properties of $F$-rings. In what follows, $R$ always denotes a regular $F$-ring, $\bar{R}$ denotes its maximal bounded sub- $F$-ring, and $I$ denotes the set of idempotents of $R$. Latin letters denote elements of rings, and Greek letters denote real numbers. Additional definitions are

$$
\begin{aligned}
& x^{+}=x \bigvee 0, \quad x^{-}=(-x) \vee 0, \quad|x|=x^{+}+x^{-}, \\
& \bar{e}_{x}=\bigvee_{n=1}^{\infty} n|x| \wedge 1, \text { and } e_{x}=1-\bar{e}_{x} .
\end{aligned}
$$

The following properties of $F$-rings (Nakano [9, Chapters I and IV]) are used throughout:

(N 1) For every $b \in R, a=\bigvee_{\lambda \in \Delta} a_{\lambda}$ implies

$$
a \wedge b=\bigvee_{\lambda \in \Lambda}\left(a_{\lambda} \wedge b\right)
$$

and the dual statement, that is, the statement with $\vee$ 's and $\wedge$ 's replaced respectively by $\Lambda$ 's and $\vee$ 's.

(N 2) If $a=\Lambda_{\lambda \in \Lambda} a_{\lambda}$, then $a+b=\Lambda_{\lambda \in \Lambda}\left(a_{\lambda}+b\right)$ and if $a=\bigvee_{\lambda \in \Lambda} a_{\lambda}$, then $a+b=V_{\lambda \in \Lambda}\left(a_{\lambda}+b\right)$ for all $b \in R$.

(N 3) If $a=\Lambda_{\lambda \in \Lambda} a_{\lambda}$, then $a b=\Lambda_{\lambda \in \Lambda} a_{\lambda} b$, and if $a=\bigvee_{\lambda \in \Lambda} a_{\lambda}$, then $a b=V_{\lambda \in \Lambda} a_{\lambda} b$, for all $b \geqq 0$.

(N 4) For all $a \in R, a^{2} \geqq 0$.

(N 5) If $a \geqq 0$ and $b \geqq 0$, then $a \wedge b=0$ is equivalent to $a b=0$.

(N 6) $R$ is archimedian, that is, for every non-negative element $a \in R, \wedge_{n=1}^{\infty}(1 / n) a=0$.

From (N 5), an element $e \in R$ is an idempotent if and only if $e \wedge(1-e)=0$. By the methods of [8], I forms a Boolean algebra with 
respect to the lattice operations of $R$. Since for $a, b \in I, a b \leqq a \wedge b$, and by (N 3), $a \wedge b=(a \wedge b)^{2}=(a \wedge b) \wedge a b$, it follows that $a b=a \wedge b$. In addition, a further application of (N 3 ) shows that $I$ is $\sigma$-complete.

From some results in $[8$, p. 530$]$, it follows that

(N 7) $\bar{e}_{x}$ is idempotent.

In the sequel, $(P)$ will denote the ideal of $R$ generated by the subset $P$ of $R$, and $(x)$ will denote the ideal generated by $x \in R$.

3. Regular $F$-rings. This section begins with a proof of a general result concerning regular rings.

Theorem 1. Let $A$ be a commutative ring with a unit 1. $A$ is regular if and only if it has property

$(\alpha)$ For each $x \in A$ there exists an element $a_{x} \in A$ such that, first, $a_{x}^{2}=a_{x}$, second, $x a_{x}=0$, third, $x+a_{x}$ has an inverse.

If $x^{0}$ is the element postulated in the definition of regularity, then $a_{x}=1-x x^{0}$.

Proof. If $A$ has property $(\alpha)$, then there exists $y \in A$ such that $y\left(x+a_{x}\right)=1$. Therefore $x=x \cdot 1=x y\left(x+a_{x}\right)=x y x$; that is, $A$ is regular.

If $A$ is regular, then there is an element $x^{0} \in A$ such that $x x^{0} x=x$. It then follows, first, that $x x^{0}$ is an idempotent of $A$, second, that $x\left(1-x x^{0}\right)=0$, third, that $1-x x^{0}$ is idempotent, and, fourth, that

$$
\left[x+\left(1-x x^{0}\right)\right]\left[x\left(x^{0}\right)^{2}-x x^{0}+1\right]=1 .
$$

Thus with $a_{x}=1-x x^{0}, A$ has property $(\alpha)$.

Theorem 2. If $x^{0} \in R$ has the property $x x^{0} x=x$, then $x x^{0}=\bar{e}_{x}$ and $1-x x^{0}=e_{x}$.

Proof. This consists in showing that $x \bar{e}_{x}=x$ and that $\bar{e}_{x} \in(x)$. From $x \bar{e}_{x}=x$, it follows that $\left(\bar{e}_{x} x\right) x^{0}=x x^{0}$, and from $\bar{e}_{x} \in(x)$ it follows that $\bar{e}_{x}=x y$ for some $y \in A$, from which we deduce $\bar{e}_{x} x x^{0}=x y x x^{0}=x y$. Thus $\bar{e}_{x}=x x^{0}$.

To show that $\bar{e}_{x} x=x$, note that

$$
x^{+} \wedge \bar{e}_{x}=\bigvee_{n=1}^{\infty} x^{+} \wedge n|x| \wedge 1=x^{+} \wedge 1
$$

and that, by the same reasoning,

$$
x^{-} \wedge \bar{e}_{x}=x^{-} \wedge 1
$$

Since by (N 7) $\bar{e}_{x}$ is idempotent, equations (2.1) and (2.2) imply

$$
0=x^{+} \wedge \bar{e}_{x} \wedge\left(1-\bar{e}_{x}\right)=x^{+} \wedge 1 \wedge\left(1-\bar{e}_{x}\right)=x^{+} \wedge\left(1-\bar{e}_{x}\right)
$$

and, by a similar line of reasoning, it follows that 


$$
0=x^{-} \wedge\left(1-\bar{e}_{x}\right) .
$$

From (N 5), (2.3), and (2.4), it follows that $x^{+} \cdot \bar{e}_{x}=x^{+}$and $x^{-} \cdot \bar{e}_{x}=x^{-}$, and hence $x \bar{e}_{x}=x$.

Finally we consider the proposition $\bar{e}_{x} \in(x)$. Since $R$ is regular, the principal ideals $(x),(|x|),\left(x x^{0}\right)$ are all equal. By $(\mathrm{N} 4), x x^{0} \geqq 0$ and $1-x x^{0} \geqq 0$, and by $(\mathrm{N} 5),|x| \cdot\left(1-x x^{0}\right)=0$. Hence $(x)$ is closed under countable sup's (see (N 3)) and (x) is an $l$-ideal in the sense of Birkhoff $[2$, p. 222], that is, if $|y| \leqq|x|$, then $y \in(x)$. Since $n|x| \in(x)$, and since $(x)$ is an $l$-ideal, it follows that $n|x| \wedge 1 \in(x)$ and $\bigvee_{n=1}^{\infty} n|x| \wedge 1=\bar{e}_{x} \in(x)$.

COROllary. $R$ is real.

PROOF. If $x \geqq \epsilon>0$, then $n|x| \wedge 1 \geqq n \epsilon \wedge 1$ and hence $\bar{e}_{x}=1$. Since $x x^{0}=\bar{e}_{x}$, it follows (Theorem 1) that $e_{x}=1-x x^{0}=0$ and that $x^{-1}$ exists.

Corollary. If $B$ is a partially ordered subring of $R$ containing $\bar{R}$, $t^{\text {hen } B}$ is real.

Proof. If $x \in B$ and $x \geqq \epsilon>0$, then $\left(x+e_{x}\right)^{-1}=x^{-1} \in R$ and $0<x^{-1}$ $\leqq 1 / \epsilon$. Therefore $x^{-1} \in \bar{R} \subseteq B$.

4. Maximal ideals of regular $F$-rings. This section is devoted to a discussion of the relationship between the maximal (ring) ideals of $R$ and those of $\bar{R}$.

THEOREM 3. There is a one-to-one correspondence $(M \rightarrow \phi(M))$ between the maximal ideals of $R$ and those of $\bar{R}$.

Proof. Definition of $\phi$ : Since $\bar{R}$ is ring- and lattice-isomorphic to the ring of continuous functions on a compact Hausdorff space, a result of Gillman and Henriksen [4, Theorem 3.3] shows that each prime ideal $\bar{P}$ of $\bar{R}$ is contained in a unique maximal ideal of $R$. If $M$ is a maximal ideal of $R$, then $\bar{R} \cap M$ is a prime ideal of $\bar{R}$ and is contained in a unique maximal ideal $\phi(M)$ of $\bar{R}$. The mapping $\phi$ is then a single valued mapping of the maximal ideals of $R$ into the maximal ideals of $\bar{R}$.

To show $\phi$ is a mapping onto the maximal ideals of $\bar{R}$, suppose $\bar{M}$ is a maximal ideal of $\bar{R}$. Then $\bar{M} \cap I$ is a prime ideal of the Boolean algebra $I$. Since $R$ is regular and commutative, a result of Morrison [7] states that $(\bar{M} \cap I)$ is a maximal ideal of $R$. The prime ideal $(\bar{M} \cap I) \cap \bar{R}$ of $\bar{R}$ contains $\bar{M} \cap I$, so it must be contained in $\bar{M}$ $=\phi[(\bar{M} \cap I)]$.

To establish that $\phi$ is biunique, it is first necessary to show that if 
$\bar{P}$ is a prime ideal of $\bar{R}$, then either $(\bar{P})$ is equal to $R$ or it is a maximal ideal. Indeed, suppose $(\bar{P})$ is different from $R$. Let $a$ be an arbitrary element of $R$ not in $(\bar{P})$. The ideal $(a,(\bar{P}))$, generated by $a$ and $(\bar{P})$, contains $a+e_{a}$. For if $a \in(\bar{P})$, then $\bar{e}_{a} \notin(\bar{P})$ because $a \cdot \bar{e}_{a}=a$. Since $\bar{e}_{a}$ does not belong to $\bar{P}$ either, it follows that $1-\bar{e}_{a}=e_{a} \in \bar{P}$. The regularity of $R$ implies that $a+e_{a}$ possesses an inverse, so $(a,(\bar{P}))$ equals $R$ and $(\bar{P})$ is a maximal ideal. If $M_{1}$ and $M_{2}$ are maximal ideals of $R$ where $M_{1} \cap \bar{R}$ and $M_{2} \cap \bar{R}$ are both subsets of the same maximal ideal $\bar{M}$ of $\bar{R}$, then $\bar{M} \cap I=M_{1} \cap \bar{R} \cap I=M_{2} \cap \bar{R} \cap I$ is a prime ideal of $I$. Therefore from [7] it follows that $M_{1}=M_{2}=(\bar{M} \cap I)$.

As in the case of the ring of all continuous functions on a completely regular space (see [6] for example), there is no guarantee that all maximal ideals of $R$ are real. Real maximal ideals are characterized by the following theorem.

THEOREM 4. A necessary and sufficient condition for a maximal ideal $M$ of $R$ to be real is that $M \cap \bar{R}$ be a maximal ideal of $\bar{R}$.

Proof. If $M$ is a real maximal ideal of $R$, then

$$
R-M \supseteq[\bar{R}+M]-M \cong \bar{R}-M \cap \bar{R}
$$

by the second homomorphism theorem for rings. The left-hand member is isomorphic to the real field and the right-hand member contains a field isomorphic to the real field. Therefore $\bar{R}-M \cap \bar{R}$ is isomorphic to the real field; hence $M \cap \bar{R}$ is a maximal ideal.

Let $M$ be a maximal ideal of $R$. If $M \cap \bar{R}$ is a maximal ideal of $\bar{R}$, then formula (4.1) implies that $[\bar{R}+M]-M$ is isomorphic to the real field.

In order to finish the proof, it suffices to show that $\bar{R}+M=R$. The following inequality can be proved for each pair of real numbers $\lambda<\mu$ and each $x \in R$, using the fact that $\left\{e_{(x-\lambda)}+\right\}$ is a spectral decomposition of 1 relative to $x[2$, p. 251] and that $x y=0$ implies $e_{x} y=y$. If $e_{x}(\lambda, \mu)$ stands for $e_{(x-\mu)^{+}}-e_{(x-\lambda)^{+}}$, then the inequality can be expressed as follows

$$
\lambda e_{x}(\lambda, \mu) \leqq x e_{x}(\lambda, \mu) \leqq \mu e_{x}(\lambda, \mu) .
$$

Therefore $x e_{x}(\lambda, \mu) \in \bar{R}$.

Let $x$ be an element of $R$ not in either $M$ or $\bar{R}$. Suppose $x e_{x}(\lambda, \mu)$ $\in M \cap \bar{R}$ for all $\lambda, \mu(\lambda<\mu)$. Then $e_{x}(\lambda, \mu) \in M \cap \bar{R}$ for all such $\lambda, \mu$ and in addition

$$
a=\sum_{N=1}^{\infty} \frac{1}{N^{2}} e_{x}(N-1, N)+\sum_{N=1}^{\infty} \frac{1}{N^{2}} e_{x}(-N,-N+1)
$$


belongs to $M \cap \bar{R}$ because the maximal ideals of the real Banach algebra $\bar{R}$ are norm-closed.

Since $\left\{e_{(x-\lambda)^{+}}\right\}$is a spectral decomposition,

$$
e_{x}(\lambda, \mu) \wedge e_{x}(\sigma, \tau)=e_{x}(\lambda, \mu) \cdot e_{x}(\sigma, \tau)=0
$$

if the closed intervals $[\lambda, \mu]$ and $[\sigma, \tau]$ have no more than one point in common. Therefore equation $(4.3)$ can be replaced $[9$, Theorem $5.15]$ by

$$
a=\bigvee_{N=1}^{\infty} \frac{e_{x}(N-1, N)}{N^{2}} \bigvee_{M=1}^{\infty} \frac{e_{x}(-M,-M+1)}{M^{2}}
$$

Now

$$
\begin{aligned}
\bar{e}_{a} & =\bigvee_{n=1}^{\infty} n a \wedge 1 \\
& =\bigvee_{n=1}^{\infty}\left[\bigvee_{N=1}^{\infty} \frac{n}{N^{2}} e_{x}(N-1, N) \bigvee \bigvee_{M=1}^{\infty} \frac{n}{M^{2}} e_{x}(-M,-M+1)\right] \wedge 1 \\
& =\bigvee_{n, N, M}\left[\frac{n}{N^{2} M^{2}} e_{x}(N-1, N) \bigvee e_{x}(-M,-M+1)\right] \wedge 1 \\
& =\bigvee_{N, M}\left\{\bigvee \frac{}{n}\left[\frac{n}{N^{2} M^{2}} e_{x}(N-1, N) \bigvee e_{x}(-M,-M+1)\right] \wedge 1\right\}
\end{aligned}
$$

and because $e=e^{2}$ implies $n e \bigwedge 1=e$ for all $n \geqq 1$, it follows that

$$
\begin{aligned}
\bar{e}_{a} & =\bigvee_{N, M}\left[e_{x}(N-1, N) \bigvee e_{x}(-M,-M+1)\right] \\
& =1 .
\end{aligned}
$$

Therefore $e_{a}=0$ and $a^{-1}$ belongs to $R$. Since this is impossible because $a \in M$, the supposition that $e_{x}(\lambda, \mu) \in M$ for all pairs $(\lambda, \mu)$ is incorrect.

Let $\lambda$ and $\mu$ be numbers such that $e_{x}(\lambda, \mu) \notin M$. Then $1-e_{x}(\lambda, \mu)$ $\in M \cap \bar{R}$. For any $x \in R$, the element $x e_{x}(\lambda, \mu)$ belongs to $\bar{R}$, and $x-x e_{x}(\lambda, \mu)$ belongs to $M$. Thus $R=\bar{R}+M$, which concludes the proof.

Closed maximal ideals figure importantly in what follows. We therefore conclude this section with a few facts about them.

TheOREM 5. The closed maximal ideals of $R$ are real.

Proof. In order to show that a closed maximal ideal $M$ is real, it suffices to show that $R-M$ is the real field. In the course of the proof of Theorem 2, it was shown that $(x)$, and hence any maximal ideal $M$ of $R$, is an $l$-ideal. Thus the quotient space $R-M$ is an $l$-group 
[2, pp. 214, 222]. If $a(M)$ stands for the image of $a \in R$ under the natural homomorphism of $R$ onto $R-M$, then $a^{+}(M) \cdot a^{-}(M)=0$. Since $R-M$ is a field, either $a^{+}(M)=0$ or $a^{-}(M)=0$; hence $R-M$ is simply ordered. We know that $R-M$ is an ordered field because the following statement is a trivial consequence of the definition of order in $R-M: a(M) \geqq 0$ and $b(M) \geqq 0$ imply $a(M) \cdot b(M)=0$.

That $R-M$ is a $\sigma$-complete vector lattice follows from the hypothesis that $M$ is a closed maximal ideal. Under these circumstances, $R-M$ is archimedian (N 6) and hence it is isomorphic to the real field [2, Ex. 2, p. 229].

THEOREM 6. There is a one-to-one correspondence between the closed maximal ideals of $R$ and those of $\bar{R}$.

Proof. If $M$ is a closed ideal of $R$, then $\phi(M)=M \cap \bar{R}$ is a maximal ideal of $\bar{R}$ (Theorems 4 and 5). The ideal $M \cap \bar{R}$ is also easily seen to be closed.

If, on the other hand, $\bar{M}$ is a closed maximal ideal of $\bar{R}$, then $\bar{M} \cap I$ is a $\sigma$-prime ideal of the Boolean algebra $I$, that is, $\bar{M} \cap I$ is a prime ideal of $I$ satisfying the added property: if $a_{n} \in \bar{M} \cap I$ for all integers $n \geqq 1$ and $a=\bigvee_{n=1}^{\infty} a_{n} \in I$, then $a \in \bar{M} \cap I$. The ideal $(\bar{M} \cap I)$ of $R$ is, by Morrison's Theorem [7], a maximal ideal. In addition, $(\bar{M} \cap I) \cap \bar{R}$ $=\bar{M}$.

To finish the proof it suffices to show that $(\bar{M} \cap I)$ is a closed ideal of $R$. Suppose $x_{n} \in(\bar{M} \cap I)$ for $n \geqq 1$ and suppose $x=\bigvee_{n=1}^{\infty} x_{n}$ belongs to $R$. Then $\bar{e}_{x_{n}} \in \bar{M} \cap I$ for $n \geqq 1$ and because $\bar{e}_{x_{n}=}^{+<} \bar{e}_{x_{n}}$ (easily verifiable),

$$
\bigvee_{m=1}^{\infty} \bar{e} x_{m}^{+}=\bigvee_{m=1}^{\infty} \bigvee_{n=1}^{\infty} n x_{m}^{+} \wedge 1=\bigvee_{n=1}^{\infty} n\left(\bigvee_{m=1}^{\infty} x_{m}^{+}\right) \wedge 1
$$

Therefore $\bar{e}_{x}+\in \bar{M} \cap I$, and also $x^{+} \in(\bar{M} \cap I)$. That $x_{n}^{-}$for $n \geqq 1$ and $x^{-}=\bigwedge_{n=1}^{\infty} x_{n}^{-}$all belong to $(\bar{M} \cap I)$ follows because $(\bar{M} \cap I)$ is an $l$-ideal. Thus $x^{+}-x^{-}=x \in(\bar{M} \cap I)$; hence $(\bar{M} \cap I)$ is closed.

5. Representation theorems for certain $F$-rings. It is clear that the ring of all $(\Phi, \mathfrak{l})$-measurable functions is a regular $M$-ring. Indeed, each point $\xi \in \Phi$ corresponds to the closed maximal ideal of all functions vanishing at $\xi$, so the only function common to all closed maximal ideals is the zero function. The ring of all $(\Phi, \mathfrak{l})$-measurable functions contains every $F$-ring of $(\Phi, \mathfrak{l})$-measurable functions. In this section it is shown that, conversely, every regular $M$-ring is ringand lattice-isomorphic to the $M$-ring of all $(\Phi, \&)$-measurable functions for a certain well defined pair $(\Phi, \mathfrak{R})$.

In the remainder of $\S 5, B$ is used to denote a real $M$-ring, $\Omega$ to 
denote the set of all real closed maximal ideals $M$ of $B$, and $J$ to denote the Boolean algebra of idempotents of $B$. If $M \in \Omega$, then $x(M)$ stands for the image of $x$ under the natural homomorphism of $B$ onto $B-M$. The symbol $x(\cdot)$ represents the real valued function defined on $\Omega$ which takes the value $x(M)$ at the point $M \in \Omega$. For each $e \in J$ consider the subset $U(e)=\{M \mid e(M)=1\}$. $\mathfrak{A}$ is used to denote the collection of all such subsets.

Lemma. $\mathfrak{A}$ is a $\sigma$-algebra and is isomorphic to $J$.

Proof. If $M$ is a closed ideal, then $M \cap J$ is a $\sigma$-prime ideal of $J$ and if $\bigcap_{\Omega} M=0$, then $\bigcap_{\Omega} M \cap J=0$ also. By a result of Sikorski [11, Theorem 1.3], $\mathfrak{A}$ is a $\sigma$-algebra and is isomorphic to the $\sigma$-complete Boolean algebra $J$.

Now we may use $R(\Omega, \mathfrak{A})$ to denote the $M$-ring of all $(\Omega, \mathfrak{A})$ measurable functions and $\bar{R}(\Omega, \mathfrak{A})$ to denote the $M$-ring of all bounded $(\Omega, \mathfrak{A})$-measurable functions.

THEOREM 7. $B$ is ring-and lattice-isomorphic to $B(\Omega, \mathfrak{A})$, an $M$-ring of $(\Omega, \mathfrak{A})$-measurable functions.

Proof. First, by the standard (Gelfand [3]) argument, $B$ can be shown to be ring-isomorphic to a ring $B(\Omega)$ of real valued functions defined on $\Omega$.

The mapping $x \rightarrow x(M)$ of $B$ onto $B-M$ preserves order. Indeed, suppose $x \geqq 0$ and $x(M) \leqq 0$. Then $x-x(M) \geqq-x(M) \geqq 0$, and, because $B$ is real, $x-x(M)$ has an inverse. However, $x-x(M)$ belongs to $M$. Therefore $x \geqq 0$ is a sufficient condition for $x(M) \geqq 0$; hence the mapping preserves order.

Define $x(\cdot) \geqq y(\cdot)$ if $x(M) \geqq y(M)$ for each $M \in \Omega$. This definition induces a partial order on $B(\Omega)$, and with $B(\Omega)$ thus ordered, the isomorphism mentioned in the first paragraph of this proof preserves order. Necessarily the lattice structure is preserved as well; hence $B$ is ring- and lattice-isomorphic to $B(\Omega)$.

Finally, each $x(\cdot) \in B(\Omega)$ is a $(\Omega, \mathfrak{A})$-measurable function. Indeed, each $M \in \Omega$ is an $l$-ideal as well as a real closed maximal ideal. (To see that this is so, look at the image of $M$ in $B(\Omega)$.) From this, it follows that $x \in M$ if and only if $\bar{e}_{x} \in M$. Therefore

$$
\begin{aligned}
U\left[e_{(x-\lambda)^{+}}\right] & =\left\{M \mid e_{(x-\lambda)^{+}}(M)=1\right\} \\
& =\left\{M \mid(x-\lambda)^{+}(M)=0\right\} \\
& =\{M \mid x(M) \leqq \lambda\}
\end{aligned}
$$

belongs to $\mathfrak{A}$ for each $\lambda$. Thus the symbol $B(\Omega)$ may be meaningfully replaced by the symbol $B(\Omega, \mathfrak{A})$ and the theorem is proved. 
It should be remarked at this point that Theorem 7 is still valid when $\Omega$ is replaced by any subset $\Omega^{*}$ where the intersection of all ideals in $\Omega^{*}$ is the zero ideal. $\mathfrak{A}$ is defined in the same manner with respect to $\Omega^{*}$ as it was with respect to $\Omega$.

The following theorem is closely related to Theorem 7 .

THEOREM 7A. The following statements are equivalent. (i) $R=\bar{R}$. (ii) $R$ is the F-ring of all ordered $n$-tuples of real numbers for some fixed integer $n$. (iii) All maximal ideals of $R$ are closed.

Proof. (i) implies (ii). Since $R=\bar{R}$ is a regular real semisimple commutative Banach algebra, it is finite dimensional [1, Theorem 3.5] and it has a representation as a ring of functions. The result follows because the number of maximal ideals of $R$ is then finite.

(ii) implies (i) and (ii) implies (iii) are trivial.

(iii) implies (ii). If all maximal ideals of $R$ are closed, then the mapping $M \rightarrow M \cap \bar{R}$ is a one-to-one correspondence between the maximal ideals of $R$ and those of $\bar{R}$ (Theorems 4, 5, and 6), and in addition each maximal ideal of $\bar{R}$ is closed (Theorem 6).

Since every maximal ideal $M$ of $R$ is closed, it follows easily that $x \in M$ if and only if $e_{x} \notin M$. Hence if $x \in \bar{R}, x+e_{x}$ belongs to no maximal ideal of $\bar{R}$. Therefore $x+e_{x}$ has an inverse in $\bar{R}$; so $\bar{R}$ is regular (Theorem 1). By an argument similar to that used in the first paragraph of the proof, $\bar{R}$ is finite dimensional. From the one-to-one correspondence $M \rightarrow M \cap \bar{R}$, we deduce that $R$ is also finite dimensional, and therefore, by Theorem 7 , Statement (ii) follows.

Definition. An algebra $A$ of $(\Phi, \mathfrak{R})$-measurable functions is $\sigma$ convex provided that $y(\cdot) \in A$ if $y(\cdot)$ is $(\Phi, \&)$-measurable and $0 \leqq y(\cdot) \leqq x(\cdot)$ where $x(\cdot)$ belongs to $A$.

Such a $\sigma$-convex algebra is necessarily a real $M$-ring; the following theorem is a converse to this statement.

\section{TheOREM 8. $B(\Omega, \mathfrak{U})$ (defined in Theorem 7 ) is a $\sigma$-convex algebra.}

Proof. In order to show $B(\Omega, \mathfrak{A})$ is $\sigma$-convex, it is first necessary to show $\bar{R}(\Omega, \mathfrak{A}) \subseteq B(\Omega, \mathfrak{U})$. It should be noted that since a lattice isomorphism preserves sup's and inf's whenever they occur, the definition of order in $B(\Omega, \mathfrak{A})$ implies that the sup in $B(\Omega, \mathfrak{A})$ is the pointwise sup. Since $B(\Omega, \mathfrak{U})$ contains all simple functions (finite linear combinations of characteristic functions of sets in $\mathfrak{A}$ ), and since every bounded measurable function is the pointwise sup of a countable set of simple functions, it follows from the conditional $\sigma$-completeness of $B(\Omega, \mathfrak{A})$ that $\bar{R}(\Omega, \mathfrak{A})$ is a sub- $M$-ring of $B(\Omega, \mathfrak{A})$.

To finish the proof, suppose that $y(\cdot) \geqq 0$ is a $(\Omega, \mathfrak{A})$-measurable function and that $y(\cdot) \leqq x(\cdot) \in B(\Omega, \mathfrak{A})$. Let $y_{n}(\cdot)=y(\cdot) \chi\{M \mid y(M)$ 
$\leqq n\}$ for integers $n \geqq 1$. Each element $y_{n}(\cdot)$ is bounded; hence it belongs to $B(\Omega, \mathfrak{A})$. In addition, $y_{n}(\cdot) \leqq x(\cdot)$ for each $n$. Therefore since $B(\Omega, \mathfrak{A})$ is an $M$-ring, $y(\cdot)=\mathrm{V}_{n=1}^{\infty} y_{n}(\cdot)$ also belongs to $B(\Omega, \mathfrak{A})$.

Corollary. If $B$ is regular, then $B(\Omega, \mathfrak{H})=R(\Omega, \mathfrak{A})$.

Proof. Suppose $0 \leqq x(\cdot)$ is an $(\Omega, \mathfrak{A})$-measurable function. Then $x(\cdot)+1$ is also $(\Omega, \mathfrak{A})$-measurable and $y(\cdot)=(x(\cdot)+1)^{-1}$ is both measurable and bounded. By Theorem $8, y(\cdot) \in B(\Omega, \mathfrak{A})$, and since $y(M) \neq 0$ for all $M \in \Omega$, it follows that the characteristic function $\chi\{M \mid y(M)=0\}=0$ and if $y$ is the isomorphic copy in $B$ of $y(\cdot)$, then the isomorphic copy $e_{y}$ of the characteristic function $\chi\{M \mid y(M)=0\}$ is zero. Therefore, $y^{-1}$ exists in $B$, and $y^{-1}(\cdot)=x(\cdot)+1 \in B(\Omega, \mathfrak{U})$. Thus $x(\cdot) \in B(\Omega, \mathfrak{A})$, and the corollary follows from this.

An $F$-ring $A$ is atomic if its Boolean algebra of idempotents is an atomic Boolean algebra. We conclude with two theorems about atomic $F$-rings.

Theorem 9. If $R$ is atomic, then $R$ is an M-ring.

PROOF. In the course of the proof of Theorem 6, it was shown that there is a one-to-one correspondence $M \rightarrow M \cap I$ between the set of closed maximal ideals of $R$ and the set of $\sigma$-prime ideals of $I$. Sikorski [11, Theorem 1.8] has shown that if a $\sigma$-complete Boolean algebra is atomic, then the intersection of its $\sigma$-prime ideals is zero. Since $x \in M$ if and only if $\bar{e}_{x} \in M \cap I$, it follows that

$$
\bigcap_{M \in \Omega} M=\bigcap_{M \in \Omega} M \cap I=0 ;
$$

hence $R$ is an $M$-ring.

An $F$-ring $A$ is complete if any arbitrary set of elements of $A$, bounded above by an element of $A$, has a least upper bound.

TheOREм 10. If $R$ is both complete and atomic, then it is a direct sum of real fields.

Proof. To show that $R$ is a direct sum of real fields, it suffices to show that $R$ is isomorphic to the ring of all real-valued functions on some space $\Omega^{*}$. Let $\Omega^{*}$ be the set of maximal ideals $M_{a}=\{x \mid x a=0\}$ where $a$ is an atom of $I$. Each ideal $M_{a}$ is closed and since $\bigcap_{\Omega} * M_{a} \cap I$ $=0$, we deduce that $\cap_{\Omega} * M_{a}=0$ also (see proof of Theorem 10).

By the remark following the proof of Theorem $7, R$ is isomorphic to $R\left(\Omega^{*}, \mathfrak{H}\right)$ ( $\mathfrak{A}$ is defined relative to $I$ and, by Lemma, is isomorphic to $I)$. The complete atomic character of $R$ insures that $\mathfrak{A}$ is the Boolean algebra of all subsets of $\Omega^{*}$. Hence $R\left(\Omega^{*}, \mathfrak{A}\right)$ is the $M$-ring of all real functions on $\Omega^{*}$. 


\section{REFERENCES}

1. R. F. Arens and I. Kaplansky, Topological representations of algebras, Trans. Amer. Math. Soc. vol. 63 (1948) pp. 457-481.

2. G. Birkhoff, Lattice theory, New York, Amer. Math. Soc. Colloquium Publications, vol. $25,1948$.

3. I. Gelfand, Normierte Ringe, Rec. Math. (Mat. Sbornik) N.S. vol. 9 (51) (1941) pp. 3-24.

4. L. Gillman and M. Henriksen, Concerning rings of continuous functions, Trans. Amer. Math. Soc. vol. 77 (1954) pp. 340-362.

5. L. Gillman, M. Henriksen, and M. Jerison, On a theorem of Gelfand and Kolmogorof concerning maximal ideals in rings of continuous functions. Proc. Amer. Math. Soc. vol. 5 (1954) pp. 447-455.

6. E. Hewitt, Rings of real-valued continuous functions I, Trans. Amer. Math. Soc. vol. 64 (1948) pp. 45-99.

7. D. R. Morrison, Bi-regular rings and the ideal lattice isomorphisms, Proc. Amer. Math. Soc. vol. 6 (1955) pp. 46-49.

8. S. Kakutani, Concrete representation of abstract $L$-spaces and the mean ergodic theorem, Ann. of Math. vol. 42 (1941) pp. 523-537.

9. H. Nakano, Modern spectral theory, Tokyo Mathematical Book, Series 2, Tokyo, 1950.

10. J. von Neumann, Regular rings, Proc. Nat. Acad. Sci. U.S.A. vol. 22 (1936) pp. 707-713.

11. R. Sikorski, On the representation of Boolean algebras as fields of sets, Fund. Math. vol. 35 (1948) pp. 247-258.

University of British Columbia 